\title{
Developing a Collaborative Learning Environment Using Web Services Techniques
}

\author{
Amal Al-Abri1 ${ }^{*}$, Zuhoor Al-Khanjari ${ }^{1}$, Yassine Jamoussi ${ }^{1,2}$, and Naoufel Kraiem ${ }^{1,2}$ \\ ${ }^{1}$ Department of Computer Science, College of Science, Sultan Qaboos University, PO Box 36, Al-Khoudh 123, \\ Muscat, Sultanate of Oman. \\ 2 RIADI Lab, ENSI, Campus of Manouba, Tunisia.
}

*Corresponding author. Email: p010844@student.squ.edu.om; \{yessine, naoufel, zuhoor\}@squ.edu.om Manuscript submitted May 9, 2016; accepted July 12, 2016.

doi: 10.17706/jsw.11.9.870-882

\begin{abstract}
Collaborative e-learning has a set of characteristics which requires interaction and negotiation among learners and teachers. It also needs support to achieve high-level requirements for learning content and systems such as accessibility, reusability, interoperability and adaptability. The focus of this paper is to investigate three commonly used standards in e-learning which are: SCORM, IMS-LD, IEEE and LOM to enhance the characteristics of collaborative e-learning in relation to the features of these standards. Due the lack of collaboration features in these standards, this paper highlights Service-Oriented Architecture (SOA) features to fulfill the collaborative e-learning requirements.
\end{abstract}

Keywords: Collaborative e-learning characteristics, e-learning standards, SOA.

\section{Introduction}

Collaborative e-learning is not simply a word called "collaborative" attached to the e-learning environment phenomena. It is much more than that. Collaborative e-learning environments can be described as a context where the computer or information technology facilitates interaction among learners for acquisition or sharing of knowledge [1] and accessing e-learning content from different systems [2]. Educators and researchers are striving to find mechanisms and tools to adapt this context in the learning process. Consequently, issues related to sharing and the ability to integrate with other components [3] like other e-learning systems or web 2.0 tools emanated.

Since collaborative e-learning is a style of e-learning environments, it requires standardization techniques to be able to fit in the web-based world. Up to now, there are over 40 standards and specifications proposed by more than a dozen international organizations [4] addressing the e-learning standardization issues. Therefore, the question is which standard or specification best addresses collaborative e-learning characteristics. This study aims to investigate how well the existing standards can effectively fit with the features of collaborative e-learning. It also provides recommendations for the selections. To limit the scope of the study, this paper is focusing on three commonly used standards in the field of e-learning.

The paper will start by giving an overview of collaborative e-learning environments and their characteristics in Section 2. Then, in Section 3, a discussion ensues about standardization in e-learning focusing on three commonly used standards (SCORM, IMS-LD, IEEE LOM) followed by a mechanism to 
match the features of these standards in relation to the characteristics of collaborative e-learning. The paper will present an overview of SOA usage for collaborative e-learning as a recommendation in Section 4. Related work in the field of using SOA for e-learning is giving in Section 5. After that, Section 6 will present a methodology for SOA implementation for collaborative e-learning. The paper will be concluded in Section 7.

\section{Collaborative e-Learning}

According to [5], collaborative learning could be described as "a variety of educational practices in which interactions among peers constitute the most important factor in learning, although without excluding other factors, such as the learning material and interactions with teachers". In lifelong education, collaborative learning is a key paradigm in informal learning (e.g. sharing knowledge among communities of practices), but has been somewhat underutilized in corporate training.

Collaborative learning focuses on providing a shared workplace for students to interact and learn through cooperation [6]. The interaction could be formed in many ways like: interactions with members of the groups they are assigned to (assigned group); interactions with members of the larger community that is the class (class group); and by interactions with peers from the discipline's community (discipline community) [7].

The main characteristics of collaborative e-learning, as suggested by [7] are:

- Active Learning: Learners participating in a constructive and iterative process of interaction and negotiation in a problem-solving task. This interaction requires learners to create, access, share and reuse learning contents, or even gathering them from different sources [8]. In addition, for sharing knowledge and resources, the interoperability feature is required.

- Group Participation: Groups of two or more learners socially interacting and collaborating through asking questions, justifying opinions, listening to others, and through negotiation, reaching a consensual answer.

- $\quad$ Role of the Instructor: The instructor provides a task to be completed, and offers qualified guidance when required. Basically, the instructor plays the role of facilitating the learning process and comments on the progress of the learners who are generating their knowledge and learning contents.

- Learner Diversity: Diversity in a group (because of learner's background), allows learners to draw different perspectives on task-related information, drawing richer interpretations and devising more complex solutions. This diversity is classified in two terms which are demographic (surface-level diversity) and learning styles, experiences and knowledge (deep-level diversity). Although the nature of collaborative-learning is based on group learning, the characteristics of a group of learners should be modeled and adapted based on the individuals (personalization), in relation to the assessment of shared knowledge which might determine the group dynamics [9].

- Learner Relationships: Relationships are expanded from instructor-to-learner, to include learner-tolearner, and learner-to-instructor relationships, where learning is multidirectional.

- Social interaction: collaborative e-learning is a social interaction between peers and teachers to facilitate knowledge sharing and discussions. This interaction could be either in a synchronous or asynchronous mood.

In order to fulfill the above identified characteristics, it is very important to select the appropriate standards and specifications to be adapted when developing collaborative e-learning environments. Aiming to address this issue, the following section will give detailed information about three common standards which are dealing with high-level requirements for learning content and systems. These standards are SCORM, IMS-LD, and IEEE LOM. The information will also include a discussion of a matching mechanism between the features of these standards in relation to the characteristics of collaborative e-learning. 


\section{Standardization}

According to ISO, a standard is a document that provides requirements, specifications, guidelines or characteristics that can be used consistently to ensure that materials, products, processes and services are fit for their purpose. The concept of a standard in e-learning is developed to provide fixed structures and communication protocols for e-learning objects. This enables interoperability between applications, by providing uniform communication guidelines that can be used throughout the design, development, and delivery of learning objects [10].

In general, e-learning standardization is concerned with the solution of two key problems: interoperability and reusability. Interoperability concerns the ability to work together and to communicate, and reusability concerns the possibility of exploiting a resource repeatedly in different systems [4].

In short, the use of standards promotes interoperability, reusability, accessibility and durability requirements, which are the major aspects to consider when designing adaptive and collaborative elearning system. Standardization is meant to support individuals, groups, or organizations, and to enable reusing their contents transparently without any manual intervention when migrating from one platform to another [11].

\subsection{E-Learning Standards}

There are over 40 standards and specifications proposed by more than a dozen international organizations [4]. However, this study shortens the investigation to three main standards, as will be discussed later.

In this section, we will briefly discuss three existing e-learning standards to check their ability to provide collaboration features. Those standards are SCORM, IMS-LD and IEEE LOM).

\subsubsection{Scorm}

SCORM (Shareable Content Object Reference Model) is one of the most commonly used standards in the web-based e-learning field. It is proposed by the Advanced Distributed Learning (ADL) organization. As ADL stated, SCORM is "a model that references a set of interrelated technical standards, specifications, and guidelines designed to meet high-level requirements for learning content and systems". This technical standard consists of three main related documents which are:

1) Content Aggregation Model (CAM): This document presents a model for learning objects aiming to support adaptive instruction based on factors, such as: learner objectives, preferences, performance and instructional techniques [12].

2) The Content Aggregation Model describes how the course content should be packaged, deployed, and delivered via any SCORM conformant compatible with a learning management system (LMS) [13]. The basic units in the Content Aggregation Model are Sharable Content Objects (SCOs) and Content Packages that are used to bundle content.

a) SCOs: Contain all contents for a set unit of instruction called "assets". The contents can be images, text, video or any other electronic media. The SCO might also include data related to learner progress, position in the particular unit of instruction, or other data related to the SCO [14].

3) Run-time Environment (RTE): The run-time specification also supports the adaptation of instructions via learning objects using learning objectives, preferences, performance and other techniques. In short, it controls the way Learning Management System (LMS) launches learning content and the communication process between the sharable content objects (SCOs) and LMS [13].

4) Sequencing and Navigation (SN): This model is basically for the dynamic presentation of learning content based on learner needs [12]. It defines the ability of a learner to navigate between two 
learning objects, as well as the sequence in which those learning objects are experienced by the learner. This specification promotes reusability and interoperability of learning content across LMS [13].

As mentioned above, the aim of this standard is to meet high-level requirements for learning content and systems. Those requirements as per ADL 2004 are as follow:

- Accessibility: The ability to locate and access instructional components from more than one remote location and deliver them to other locations.

- Adaptability: The ability to tailor/adapt instruction to individual and organizational needs.

- Affordability: The ability to reduce the time and costs required in delivering instruction for the purpose of increasing efficiency and productivity.

- Durability: The ability to upgrade technology evolution without the need to redesign, reconfiguration or recode.

- Interoperability: The ability to use instructional components developed in one location with one set of tools or platform, and use them in another location with a different set of tools or platform.

- Reusability: The flexibility to incorporate instructional components in multiple applications and contexts.

A summary of the SCORM specifications and the requirements is shown in Fig. 1.

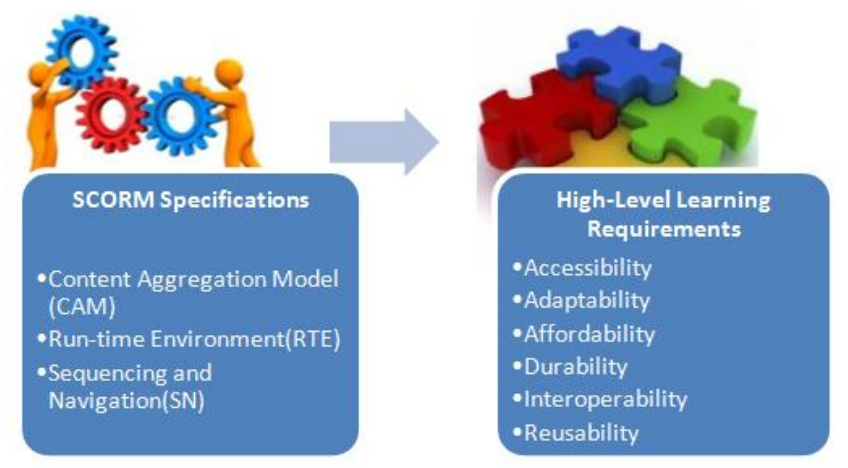

Fig. 1. SCORM specifications.

A. SCORM Features

- Able to support high-level requirements for learning (Accessibility, Adaptability, Affordability, Durability, Interoperability, Reusability)

- Provides a reference interaction model between learners and learning contents.

- Permits the sharing of learning objects between institutions [15].

- Reusability of learning objects in several learning activities [15].

- Able to track an individual's progress through a learning activity.

- Provides a mechanism for sequencing an individual's learning activities.

B. SCORM Limitations

- Unable to provide individual learners with any information about other learners who are engaged in the same learning activity

- Doesn't capture the entire picture of e-learning.

- SCORM specifications are limited to a single learner mindset.

\subsubsection{IMS-Ld}

IMS-LD (INSTRUCTIONAL MANAGEMENT SYSTEMS-LEARNING DESIGN) is a standard that was developed by the Open University of the Netherlands. IMS-LD is a meta-language that is based on the Educational Modeling Language (EML). It is a specification developed to support the use of a wide range of 
pedagogies in online learning. Rather than attempting to capture the specifics of many pedagogies, it does this by providing a generic and flexible language (IMS Global Learning Consortium, 2003). IMS-LD provides a means of expressing many different pedagogical approaches including collaborative learning [16]. This standard allows specifying formally learning units corresponding to the description of the resources and the scenario managing those units [17].

IMS LD consists of three levels of implementation and compliance (A, B, and C); with each level extending and incorporating the previous (see Fig. 2).

- Level A: This level is a series of activities (assessment, discussion, simulation), performed by one or more actors (learners, teachers, etc.). It also includes a set of roles, in an environment consisting of learning objects or services [18]. In short, Level A contains all the vocabulary needed to support pedagogical diversity [13].

- Level B: This level plays a support specification by adding Properties (storing information about a person or group), Conditions (placing constraints upon flow), monitoring services and global elements to level A [18]. As a result, this enables personalization, adaptation, sequencing and feedback [13].

- Level C: The role of this level is to enable the use of notifications in Level B. (Triggered events - e.g., if a student asks a question, the teacher needs to be notified that a response is needed) [18], which is generated by an outcome and cause to derive a new activity available for a role to be executed [13].

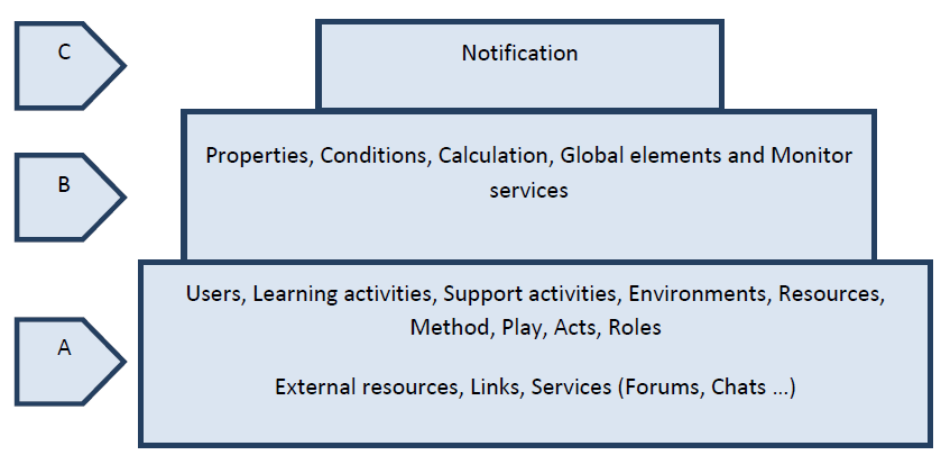

Fig. 2. The three levels specification of IMS learning design.

\section{A. IMS-LD Features}

- Enables the construct of learning design approaches into a meta-language [19].

- Focuses on the organization of learning activities [20].

- Describes and implements learning activities based on different pedagogies.

- Can support asynchronous cooperative learning.

- Can support adaptive user grouping [21].

- Supports group participation in e-courses [8].

- Reusability of learning designs and materials [8].

- Coordinates the use of learning content with collaborative services.

B. IMS-LD Limitations [22]-[24]

- Provides insufficient support to model group-based, synchronous collaborative learning activities

- Poor support for modeling varied forms of social interaction.

- Lack of support for modeling artifacts (vote, answer, argument).

- No support for modeling dynamic features.

- Cannot be easily adopted by learners (Designed with control in mind).

\subsubsection{IEEE LOM}

LOM (LEARNING OBJECT METADATA) is an e-learning standard developed by IEEE (Institute of Electrical and Electronics Engineers) [13]. It is a globally well-known open standard. LOM standard specifies the syntax and semantics of learning object metadata, which may be defined as the attributes required 
describing a learning object [4]. The learning object is an entity, which is either digital or non-digital that can be used for learning, education, or training (IEEE 2002, p1).

The main purpose of LOM is to support the sharing and reusability of learning objects. Consequently, this will lead to help discoverability, and to facilitate their interoperability, usually in the context of online learning management systems (LMS) [25].

LOM is comprised of a hierarchy of around fifty descriptive metadata elements [26] grouped into nine categories which are: General; Life Cycle; Meta-Metadata; Technical; Educational; Rights; Relation; Annotation; and Classification [17].

1) General: Provides information to describe the learning object (LO) as a whole.

2) Lifecycle: Provides information about the history and current status of the learning object and those who have contributed to its creation.

3) Meta-Metadata: Provides information about the metadata that describes the learning object, as opposed to the learning object itself.

4) Technical: Provides technical requirements and characteristics of the LO.

5) Educational: Contains educational and pedagogical characteristics of the LO.

6) Rights: Gives details of intellectual property rights and conditions guiding the use of the learning object.

7) Relation: Describes the relationship between the learning object and other related objects.

8) Annotation: Provides comments on the educational use of the learning objects, including when and by whom the comments were created.

9) Classification: Contains classification schemes used to describe different characteristics of the learning object.

Normally these categories group together data elements, of which there are two types:

- Aggregate elements (also described as container elements) which contain other data elements and which do not have individual values.

- Simple data elements (the "leaf" nodes of the hierarchical "tree" structure), which have individual values.

A. IEEE LOM Features

- Enables learners or instructors to search, evaluate, acquire, and utilize learning objects.

- Supports the reusability of learning objects.

- Facilitates learning objects interoperability (sharing and exchange of learning objects across LMS).

- Feasibility of tagging learning materials automatically.

- Enables the retrieval module of a retrieval system to retrieve personalized learning objects for an individual learner.

- Helps the tutoring module of a tutoring system in the tutoring processes.

- Adaptable for learning objects (application profiles).

B. IEEE LOM Limitations

- Offers little opportunity to include information on intellectual property.

- Requires more effort to create each record, and compromises between interoperability and addressing specific requirements [27].

- The standard as a whole is monolithic [27].

- Lacks the ability to describe IPR, technical, lifecycle issues, and others [27].

- Can't describe educational properties [27].

- The descriptions of LOM are context-independent and static classifications (it requires values to be assigned to 50 or more metadata attributes) [28].

- Limits of string-based indexing engines [29].

- Inequality of usage of the different data elements (20 out of 50) used [30].

\subsection{Matching Mechanism}


A matching mechanism must exist between the features of these standards in relation to the characteristics of collaborative e-learning.

Considering the general information about SCORM, IMS-LD, and IEE LOM given above, a matching mechanism between the features of these standards in relation to the characteristics of collaborative elearning is required. The following table (Table 1) presents the collaborative e-learning characteristics as parameters to check in terms of satisfying these characteristics by the three standards.

Table 1. Matching the Features of E-Learning Standards and the Characteristics of Collaborative E-Learning

\begin{tabular}{|l|l|l|l|}
\hline \multirow{2}{*}{ Characteristics of collaborative e-learning } & \multicolumn{1}{|c|}{ SCORM } & \multicolumn{1}{|c|}{ IMS-LD } & \multicolumn{1}{|c|}{ IEE LOM } \\
\cline { 2 - 4 } $\begin{array}{l}\text { Active Learning } \\
\text { (accessibility, reusability, interoperability) }\end{array}$ & $\begin{array}{l}\text { Accessibility, } \\
\text { Affordability, } \\
\text { Durability, } \\
\text { Interoperability, } \\
\text { Reusability }\end{array}$ & Reusability & $\begin{array}{l}\text { Accessibility, } \\
\text { Interoperability, } \\
\text { Reusability }\end{array}$ \\
\hline $\begin{array}{l}\text { Group Participation } \\
\text { (participation, interaction, collaboration) }\end{array}$ & $\begin{array}{l}\text { Reference } \\
\text { interaction, } \\
\text { Single learner } \\
\text { mindset }\end{array}$ & $\begin{array}{l}\text { Participation, } \\
\text { Coordinate } \\
\text { collaborative } \\
\text { services }\end{array}$ & Not applicable \\
\hline $\begin{array}{l}\text { Role of the Instructor } \\
\text { (facilitating, commenting, feedback) }\end{array}$ & $\begin{array}{l}\text { Single authority } \\
\text { (Teacher) }\end{array}$ & Feedback \\
\hline $\begin{array}{l}\text { Learner Diversity } \\
\text { (adaptability, interpretations, personalization) }\end{array}$ & Adaptability & $\begin{array}{l}\text { Adaptability, } \\
\text { personalization, } \\
\text { pedagogical } \\
\text { diversity }\end{array}$ & Adaptability, \\
personalization,
\end{tabular}

Although, IMS Learning design is more effective in supporting collaborative and adaptive learning experiences (when compared with SCORM and IEE LOM), it is mainly because it can easily be understood by both educators and students [13]. Yet, all of the above mentioned standards/specifications are lacking in fully supporting collaborative learning, as shown in Table 1. Therefore, we can conclude that, existing standards do have some provisions for collaboration, but require substantial extensions to accommodate common practice in collaborative e-learning environments.

\section{SOA for Collaborative e-Learning}

As we discussed in the previous section, the current e-learning standards are lacking certain attributes to provide collaboration and group discussion features because of their structure. Besides, collaborative learning focuses on the generation of interaction, assessment and collaboration in the team environment [31]. Therefore, in order to satisfy the characteristics of collaborative e-learning, the recommendation is to use Service-oriented architecture (SOA). This architecture offers a remarkable ability and technique to design a collaborative system [32].

Collaborative learning using SOA helps in distributing the learning content more efficiently and consequently promotes Reusability, Interoperability, Accessibility and Modularization [33]. Being webservices based, the framework is capable of facilitating the creation of a collaborative workspace [32].

According to [34], SOA is "The policies, practices, and frameworks that enable application functionality to be provided and consumed as sets of services published at a granularity relevant to the service consumer. 
Services can be invoked, published and discovered, and are abstracted away from the implementation using a single, standards-based form of interface". The architecture is a well-defined and self-contained software entity. It includes a discoverable and invokable interface to provide certain capabilities over networks using standard protocols [35].

A common set of service-oriented principles has been established to ensure the development of flexible and agile frameworks. According to [36] the principles are:

- Reusable: supports potential reuse.

- Interoperable: components are interoperable.

- Share a formal contract: Services, services users, and potential users interact through a formal contract.

- Loosely-coupled: interaction happens without the need for tight, cross service dependencies.

- Compassable: Services may compose other service.

- Autonomous: service logic resides within a specific boundary.

- Discoverable: allows the descriptions to be discoverable and understandable.

- Transparency: handles all affairs related to location search.

\section{Related Work}

In the domain of learning and education, there have been many attempts by researchers to adopt SOA in developing e-learning environments in general and collaborative e-learning in particular.

As an example, [31] proposed a framework of Knowledge Management Learning System (KMLS) for adult education, based on a learning process and a Service Oriented Architecture (SOA). The proposed framework integrated both web services and workflow technology to construct a workflow-based learning environment in order to help the facilitation of collaborative learning in an efficient and effective way.

Also, [33] proposed a collaborative learning environment using SOA which helps in distributing the learning content more efficiently and promotes reusability, interoperability, accessibility and modularization. The implementation incorporated web-service business process management (BPM) technology for the management of collaborative learning processes.

In [37], the author proposed a framework using the Web Service approach and SOA. The approach aims to increase the efficiency and effectiveness of collaborative learning in terms of Reusability, Interoperability, Accessibility and Modularization. The defined system is a 3-tier architectural e-learning system which is planned to be an open source application with client-scripting facility. It also supports the cross-browser and it is fully integrated with different databases; MS SQL Server, MS Access, Oracle, and LDAP.

Another work done by [38] using the SOA concept and web services propose a framework for virtual learning environment (VLE) systems to provide an integrated model. The model is designed to provide a flexible integration of distributed VLE systems in which all the learning services and applications are welldefined and loosely connected. In doing so, the aim was to increase the reusability, interoperability, flexibility and interactivity in heterogeneous software.

Towards online virtual resources utilization, [35] developed an extended architectural design for computer science students to access virtual resources online. The proposed approach uses SOA to support the functionality and extend the architectural design of LMS (Moodle). The services facilitating the development of software tools have been developed first as software and published in the cloud where it could be then integrated into Moodle. The approach used Learning Interoperability Tool (LTI) published by IMS Global Learning Consortium for security criterion.

A multitier software architecture for Virtual Campus (VC) based on SOA integration of several e-learning platforms has been proposed by [39]. The Virtual Campus Advanced Architecture project (VCAA) has been defined and implemented based on a set of canonical interfaces. These interfaces standardize the main functions of learning management systems (LMSs) leading to isolating the VC from e-learning platforms 
which make it easy to shift between different LMSs without changing the VC. Blackboard Learn, Moodle, and Sakai have been selected to implement these interfaces.

In order to enhance the reusability of learning resources, a mechanism for e-learning to communicate under SOA architecture proposed by [40]. The developed service registry and repository framework is aiming to facilitate the publication and discovery of teaching resources using REST services. The framework consists of three layers. Top layer provides a technique to assist the register of services by providers and the discovery of services by the consumer. Middle layer acts as an engine to handle the interaction between the first two layers and managing the information of registered services. The bottom layer includes adaptors to streamline the access to database repositories and file systems.

\section{Methodology for SOA Implementation for Collaborative e-Learning}

SOA is a structural model composed of standard components, such as web service technology. The web service application is described using Extensible Markup Language (XML). Its communication protocol is based on the Simple Object Access Protocol (SOAP) which allows any two parties/applications to communicate with each other. Each learning object provider, which is the owner of a Learning Object (LO) Service, publishes or updates its own service description using Web service definition language (WSDL) to the registry directly [34], and they can be located using a standard Universal Description, Discovery, and Integration (UDDI).

SOA defines three roles which are service provider, service requester, and service broker. The interaction between the three roles in the case of collaborative learning as shown in Fig. 3 will be as follows:

- Service provider: Service providers use a universal web services registry called UDDI to publish learning services (contents/application) to be available to the services requesters to find desired services. When the learning objects content or application are created as services, they will be described by a WSDL document to let the service requesters know how to use them [38].

- Service requester: In the collaborative learning scenario, the learners will search for information from the knowledgebase and discuss the learning tasks with peers or seek assistance from a teacher via chatting [33]. The collaborative e-learning platform (service requester) will work with the service registry to identify the reusable service(s) to be invoked and composed that satisfy the learner's needs to accomplish the learning activities. After finding the reusable services, the service requester will negotiate with the service providers to bind services [35].

- Service broker: Acts as a medium for web services. Its role is to accept registration requests from service providers and process query/service requests from service requesters.

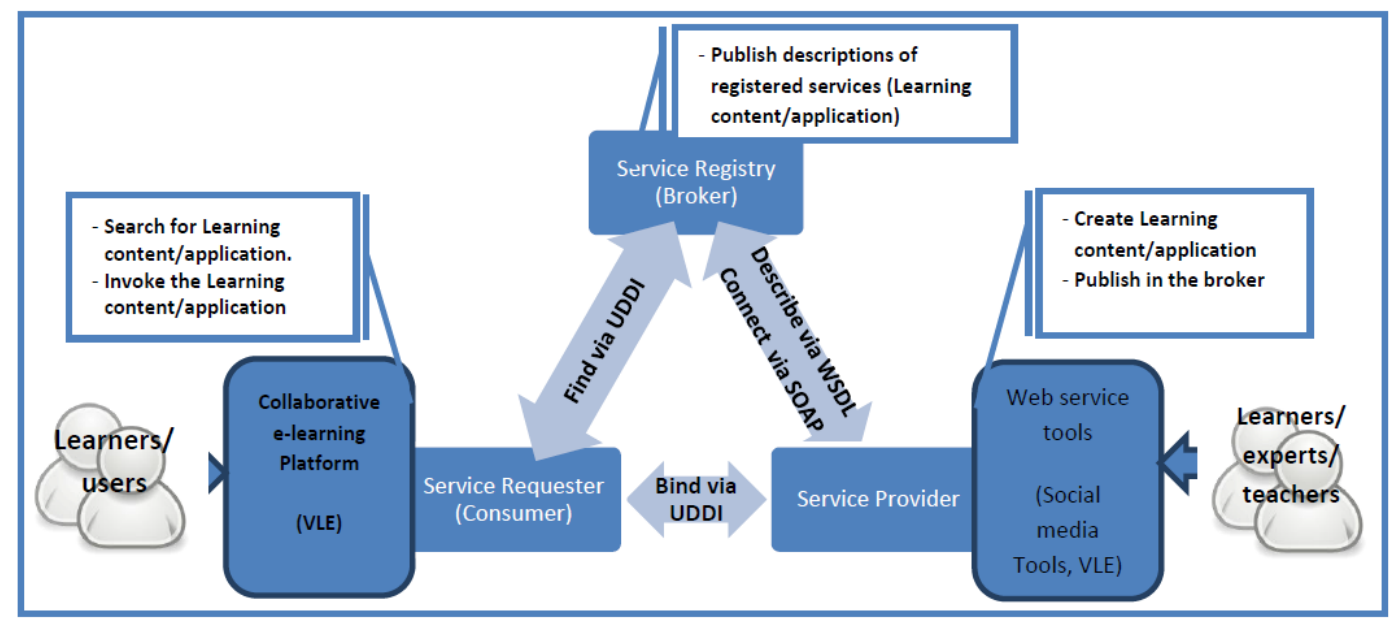

Fig. 3. Three roles of SOA architecture. 


\section{Conclusion}

This paper investigated three commonly used standards/specifications (SCORM, IMS-LD, IEEE LOM) in the field of e-learning in terms of satisfying the characteristics of collaborative e-learning. As a result, due to the structure of those standards, they are lacking certain necessary attributes to provide seamless collaboration and group modeling. Therefore as a recommendation, the study realized the effectiveness of SOA in supporting the collaboration feature integrating collaboration tools such as social media tools. The main reason for that is the structure and the standards available which mainly support the web serviceoriented platforms. Consequently, many e-learning systems specifically for collaboration have been developed based on SOA as the paper presented in the related work section. A methodology for SOA implementation has been proposed and discussed in section 6 which specifically focused on the roles of this architecture in the process of collaborative learning. However, for the implementation purpose of this methodology, a development process considering all the collaboration characteristics needs to be identified and modeled which is tagged as a future work.

\section{References}

[1] Liaw, S. S., \& Huang, H. M. (2006). Developing a collaborative e-learning system based on users' perceptions. Computer Supported Cooperative Work in Design. Springer Berlin Heidelb.

[2] Masud, M. (2016). Collaborative e-learning systems using semantic data interoperability. Computers in Human Behavior, 61, 127-135.

[3] Molinari, A. (2015). Collaboration services as a new perspective for e-learning systems. Proceedings of the Multidisciplinary Academic Conference.

[4] Llamas-Nistal, M., Caeiro-Rodríguez, M., \& Castro, M. (2011). Use of e-learning functionalities and standards: The Spanish case. IEEE Transactions on Education, 54(4), 540-549.

[5] Dillenbourg, P., Järvelä, S., \& Fischer, F. (2009). The evolution of research on computer-supported collaborative learning. Technology-enhanced learning.

[6] Li, Q., Lau, R. W., Shih, T. K., \& Li, F. W. (2008). Technology supports for distributed and collaborative learning over the internet. ACM Transactions on Internet Technology, 8(2).

[7] Doyle, C., Sammon, D., \& Neville, K. (2015). Building an evaluation framework for social media-enabled collaborative learning environments (SMECLEs). Journal of Decision Systems, 24(3), 298-317.

[8] Shariat, Z., Hashemi, S. M., \& Mohammadi, A. (2014). Research and compare standards of e-learning management system: A survey. International Journal of Information Technology and Computer Science (IJITCS), 6(2), 52.

[9] De Marsico, M., Sterbini, A., \& Temperini, M. (2013). A framework to support social-collaborative personalized e-learning. Human-Computer Interaction. Applications and Services .

[10] UȚĂ, I. A. (2007). E-learning standards. Informatica Economica Journal.

[11] Mansouri, D., Mille, A., \& Hamdi-Cherif, A. (2014). Adaptive delivery of trainings using ontologies and case-based reasoning. Arabian Journal for Science and Engineering, 39(3), 1849-1861.

[12] Advanced distributed learning (ADL) sharable content object reference model (SCORM®) 2004 2nd edition overview. Retrieved from http://www.adlnet.org/

[13] Hamdaoui, N., Idrissi, M. K., \& Bennani, S. (2014). Serious games in education towards the standardization of the teaching-learning process. Advances in Educational Technologies, 174.

[14] Lowman, D. (2004). Sharable content object reference model Retrieved from http://www.etc.edu.cn/eet/eet/articles/scorm/start.htm

[15] Paulo, Al James Uhomoibhi, (2010). Issues of e-learning standards and identity management for mobility and collaboration in higher education. Campus-Wide Information Systems. 
[16] Leo, D. H., Pérez, J. I. A., \& Dimitriadis, Y. A. (2004, August). IMS learning design support for the formalization of collaborative learning patterns. Proceedings of the IEEE International Conference on Advanced Learning Technologies (pp. 350-354).

[17] El Mhouti, A., Nasseh, A., \& Erradi, M. (2014). Towards a socioconstructivist and collaborative learning design approach to modeling pedagogical contents used in e-learning: implementation and experimentation. International Journal of Innovation and Applied Studies, 8(4), 1653.

[18] Karampiperis, P., \& Sampson, D. (2005, February). Designing learning services for open learning systems utilizing IMS learning design. Proceedings of the 4 th IASTED International Conference on WebBased Education (pp. 21-23).

[19] Dagienè, V., \& Kurilovas, E. (2015). Design of lithuanian digital library of educational resources and services: The problem of interoperability. Information Technology And Control, 36(4).

[20] Le Corre, F., Fauvel, C., Hoareau, C., Querrec, R., \& Buche, C. (2012, November). Chrysaor: An agentbased intelligent tutoring system in virtual environment. Proceedings of the International Conference on Virtual Learning (pp. 39-45).

[21] Specht, M., \& Burgos, D. (2006). Implementing Adaptive Educational Methods with IMS Learning Design.

[22] Miao, Y., Hoeksema, K., Hoppe, H. U., \& Harrer, A. (2005, May). CSCL scripts: Modelling features and potential use. International Society of the Learning Sciences.

[23] Paramythis, A. (2008, July). Adaptive support for collaborative learning with IMS learning design: Are we there yet. Proceedings of the 5th International Conference on Adaptive Hypermedia and Adaptive Web-Based Systems Workshop on Adaptive Collaboration Support.

[24] Chatti, M. A., Jarke, M., \& Specht, M. (2010). The 3P learning model. Journal of Educational Technology \& Society, 13(4), 74-85.

[25] Sicilia, M. A., Sanchez-Alonso, S., Garcia-Barriocanal, E., Minguillón, J., \& Rajabi, E. (2013, April). Exploring the keyword space in large learning resource aggregations: The case of GLOBE. In Workshop on learning object analytics for collections, repositories and federations.

[26] Tasso, S., Pallottelli, S., Rui, M., \& Laganá, A. (2014). Learning objects efficient handling in a federation of science distributed repositories. Computational Science and Its Applications.

[27] Phil, B. (2015). LRMI, Learning resource metadata on the web. Retrieved from http://blogs.pjjk.net/phil/lrmi-learning-resource-metadata-on-the-web-from-the-lile2015-workshop/

[28] Allert, H., Dhraief, H., \& Nejdl, W. (2002). How are learning objects used in learning processes? instructional roles of learning objects in LOM. World Conference on Educational Multimedia, Hypermedia and Telecommunications.

[29] Motelet, O., \& Baloian, N. A. (2005, January). Taking advantage of LOM semantics for supporting lesson authoring. On the Move to Meaningful Internet Systems.

[30] Ochoa, X., Klerkx, J., Vandeputte, B., \& Duval, E. (2011). On the use of learning object metadata: The globe experience. Towards Ubiquitous Learning.

[31] Chang, W. J., Chen, H. H., Chu, Y. S., Chen, K. J., \& Chen, M. J. (2006, October). Application of knowledge management learning system to adult collaborative learning based on service-oriented architecture. Proceedings of the 36th Annual Conference on Frontiers in Education (pp. 9-14).

[32] Rong, Z. J., Ying, B. S., \& Dan, B. B. (2010, August). A service oriented architecture for collaborative product design. Applied Mechanics and Materials .

[33] Fang, C. F., \& Sing, L. C. (2009). Collaborative learning using service-oriented architecture: A framework design. Knowledge-Based Systems, 22(4), 271-274.

[34] El-Bakry, H. M., \& Mastorakis, N. (2009). Advanced technology for e-learning development. Proceedings of the Recent Advances in Applied Mathematics and Computational and Information Sciences. 
[35] Al-Khanjari, Z., Al-Roshdi, Y., \& Kraiem, N. (2014). Virt-res: Developing extended architectural design for computer science virtual resources using SOA. International Journal of Software Engineering and Its Applications, 8(9), 125-136.

[36] Lozano, G. (2011). A traceability model for management of service-oriented landscapes. Master thesis.

[37] Jabr, M. A., \& Al-Omari, K. H. (2010). Design and implementation of e-learning management system using service oriented architecture. World Academy of Science, Engineering and Technology, 64, 59-64.

[38] Abdelhag, M. E., \& Osman, S. E. F. (2014). SOA for effective data integration of virtual learning environment systems. International Journal of Advanced Research in Computer Science and Software Engineering, 4(6), 680-685.

[39] Huertas, F., \& Navarro, A. (2015). SOA support to virtual campus advanced architectures: The VCAA canonical interfaces. Computer Standards and Interfaces, 40, 1-14.

[40] Wijayanayake, J. I. (2016). REST based registry framework for service oriented e-learning. International Journal of Computer and Information Technology, 5(1), 10-17.

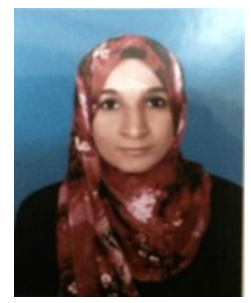

Amal Bati Said Al-Abri is currently a PhD candidate in the Department of Computer Science, College of Science at Sultan Qaboos University, Sultanate of Oman. She received her MSc in information technology from Loughborough University, UK. She worked as a lecturer for more than 9 years at Ibri college of Technology in Oman.

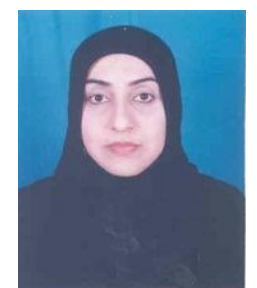

Zuhoor Abdullah Salim Al-Khanjari is the HOD of the Department of Computer Science, College of Science at Sultan Qaboos University, Sultanate of Oman. She is an associate professor in software engineering. She received her BSc in mathematics and computing from Sultan Qaboos University, Sultanate of Oman, MSc and PhD in computer science (software engineering) from the University of Liverpool, UK. Her research interests include software engineering, software testing techniques, database management, e-learning, mlearning and mobile computing. Currently, she is the coordinator of the software engineering group in the Department of Computer Science, Sultan Qaboos University, Sultanate of Oman. Also, she is coordinating elearning facilities in the same department. She is a member in the editorial board of the International Arab Journal of Information Technology (IAJIT) and a member in the executive committee of the International Arab Conference on Information Technology (ACIT).

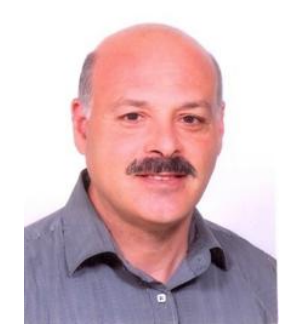

Yassine Jamoussi is a HDR between University of Manouba - University of Toulouse France). He is currently assistant professor of computer science at Sultan Qaboos University. His research interests include process-centered environment, modeling, and meta-modeling of flexible process, process enactment, modeling variability, adaptation, evaluation of process model, service oriented architecture. His research work has been supported by several funding, such as CMCU (Europeen), INRIA (French), MRT (Ministry of Research and Technology and Industry of Tunisia), IG (SQU Oman). He has been invited to present his research in many countries in North America, Europe, Africa and in the Middle East. Dr Yassine Jamoussi has been conference chair of IEEE conferences and member of over 20 program committees. He published 20 Journal and 40 conference papers in International journals and conference proceedings. 


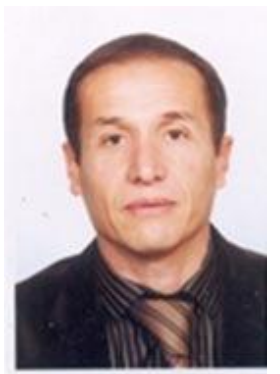

Naoufel Kraiem is currently an associate professor of computer science at SQU. His research interests include IT adoption and usage information modelling, software engineering, software product lines and CASE tools. His research work has been supported by funding of the CNRS, INRIA, MRT (Ministry of Research and Technology and Industry) and by the Commission of the European Communities under the ESPRIT Programmes (BUSINESS CLASS). He has had several articles published in many journals such as a management science information systems research communications of the ACM and IEEE Transactions. He has been invited to present his research in many countries in North America, Europe, Africa and in the Middle East. Pr Naoufel Kraiem has been a member of over 20program committees. 